\title{
A review on current nanomaterials and their drug conjugate for targeted breast cancer treatment
}

\author{
This article was published in the following Dove Press journal: \\ International Journal of Nanomedicine \\ 27 March 2017 \\ Number of times this article has been viewed
}

Joanna Jinling Lee'

Latifah Saiful Yazan ${ }^{2}$

Che Azurahanim Che

Abdullah ${ }^{3}$

'Laboratory of Molecular Biomedicine, Institute of Bioscience, ${ }^{2}$ Department of Biomedical Science, Faculty of Medicine and Health Sciences, ${ }^{3}$ Department of Physics, Faculty of Science, Universiti Putra Malaysia, Selangor, Malaysia
Correspondence: Latifah Saiful Yazan Department of Biomedical Science, Faculty of Medicine and Health Sciences, Universiti Putra Malaysia, 43400 UPM Serdang, Selangor, Malaysia

$\mathrm{Tel}+60389472308$

Fax +60389436178

Email latifahsy@upm.edu.my

\begin{abstract}
Breast cancer is the most common malignancy worldwide, especially among women, with substantial after-treatment effects. The survival rates of breast cancer have decreased over the years even with the existence of various therapeutic strategies, specifically, chemotherapy. Clinical drugs administered for breast cancer appear to be non-targeting to specific cancer sites leading to severe side effects and potentially harming healthy cells instead of just killing cancer cells. This leads to the need for designing a targeted drug delivery system. Nanomaterials, both organic and inorganic, are potential drug nanocarriers with the ability of targeting, imaging and tracking. Various types of nanomaterials have been actively researched together with their drug conjugate. In this review, we focus on selected nanomaterials, namely solid-lipid, liposomal, polymeric, magnetic nanoparticles, quantum dots, and carbon nanotubes and their drug conjugates, for breast cancer studies. Their advantages, disadvantages and previously conducted studies were highlighted.
\end{abstract}

Keywords: breast cancer, therapeutic strategies, nanomedicine, advantages, disadvantages

\section{Introduction}

Cancer is a disease characterized by an uncontrolled proliferation of cells, which can invade and spread via the lymphatic system to distant parts of the body. ${ }^{1}$ Having severe health consequences, cancer still ranks as the major cause of death worldwide. ${ }^{2}$ The research advancement of technology in the medical field has been ongoing for decades but with little significant improvements toward cancer treatment. According to the World Health Organization (WHO), the rate of deaths worldwide due to cancer is estimated to be $13 \%$, attributing 8.2 million deaths each year. With more than a hundred cancer types, the world is expected to see an increase of $70 \%$ of new global cancer incidences only within the next 2 decades. Breast cancer is the most common among all cancer types affecting females and is the leading cause of cancer mortality next to lung cancer. ${ }^{3}$ Among females, breast cancer alone accounts for $25 \%$ of all cancer cases and $15 \%$ of all cancer deaths, with an estimation of 1.7 million cases and 521,900 deaths, respectively, in $2012 .^{4}$

The major drawback of the current cancer therapy strategies is the inability to deliver specific drug to the target, causing the drug to affect both healthy and cancerous cells alike. Most drugs used in conventional therapeutic strategies have low solubility, high metabolism and are hydrophobic, making them biologically unavailable leading to systemic toxicity. ${ }^{5}$ The development of nanomedicine increases the possibility of specific targeted delivery, which overcomes tumor barriers. It is expected to better facilitate drug delivery and increase efficacy while minimizing the side effects of anticancer drugs. ${ }^{6}$ 
Nanomaterials have now become an interesting topic for research in various fields, especially in the medical area. The fact that nanomaterials being very tiny but having a broad range of application possibilities brings about promising contribution worldwide. ${ }^{7}$ Nanomedicine is among the most actively researched areas of nanotechnology, having a great impact on shaping the future for the treatment of cancer. ${ }^{8}$ It was previously reviewed that cancer nanotechnology is the application of interdisciplinary field for cancer diagnosis and treatment, focusing mainly on the design modification for biological application. ${ }^{9}$ In this review, we highlight the various nanomaterials and drugs that have been studied for targeted breast cancer treatment.

\section{Therapeutic strategies for breast cancer and their limitations}

According to the literature, the risk of breast cancer associated with personal or family history and inherited mutations in breast cancer susceptibility genes have also increased over the years. ${ }^{10}$ The treatment following early diagnosis of breast cancer generally involves surgery, hormonal therapy, radiation therapy, targeted therapy, chemotherapy or a combination of these standard therapeutic strategies. ${ }^{11}$

Surgery, also known as mastectomy, involves the removal of the breast entirely or partially depending on the stages of breast cancer. The early detection and removal of cancerous tumor prevent cancer metastases to other sites of the body. Surgery is usually combined with other therapeutic methods, and the removal of tumor can also help other treatments to work more effectively. Hormone therapy is also known as endocrine therapy, and it uses hormones to stop or slow down the growth of cancerous cells. It is commonly used to treat cancer that relies on hormones to grow, such as prostate and breast cancers. Radiation therapy is another standard therapeutic strategy where a high dose of radiation is used to kill cancer cells and shrink tumor. For breast cancer, internal radiation therapy with a solid source is commonly used. Also known as brachytherapy, this type of treatment requires radiation in the form of capsules or liquid to enter the body or near the cancer area. ${ }^{12}$

Targeted therapy involves a drug with very specific mechanism that acts on a specific target or biological pathway causing the destruction of cells. It is yet another type of cancer treatment that specifically targets cancer cells to prevent growth, division and spread. It is the foundation of precision medicine, which makes use of small-molecule drugs or monoclonal antibodies. The treatment that uses drugs to kill cancerous cells is known as chemotherapy. The drugs administered work to stop or slow down the growth of cancer cells since mutated cells grow and divide quickly. ${ }^{12}$

Standard therapeutic strategies that were developed and improved alongside with technology have indeed contributed to improving the life quality of mankind. Therapeutic strategies have enabled most patients to recover from cancer. Studies have shown that early prevention and detection of cancer while understanding the adverse effect of treatment strategies have higher cure rates. ${ }^{13}$ However, there are limitations to each of the present standard therapeutic strategies. The major concern with the current therapeutic strategies for cancer treatment is that they are treatments that are nonspecific. $^{14}$

Drugs, which are administered through various therapeutic strategies, are usually administered intravenously leading to general systemic distribution causing the drug toxicity to affect both healthy and cancerous cells. ${ }^{15}$ This causes therapeutic drugs to not only affect healthy cells but also prevent sufficient drug dosages to be delivered to the tumor site causing the treatment to deem ineffective. Cancer therapeutic drugs can be obtained from the natural plant source or produced synthetically. However, most anticancer drugs are hydrophobic in nature and lack solubility in water, leading to poor bioavailability as an effective drug for breast cancer treatment. ${ }^{16}$ The problem of poor chemical stability also affects the delivery and the rate of uptake in tumor sites hampering the dose effect. ${ }^{2}$ Furthermore, standard cancer chemotherapeutic treatments are limited in their solubility, selectivity toward tumor sites and are multidrugresistant (MDR) increasing the resistance of tumor against anticancer drugs. ${ }^{15}$

\section{Nanomaterials for targeted breast cancer treatment}

Nanomaterials are engineered using different material platforms to be nanosized similar to proteins, viruses and other macromolecular structures of the biological makeup. Previous reviews have described different material platforms such as metallic, organic and semiconducting particles emerging as nanomaterials for biomedical applications, especially in cancer therapy ${ }^{17}$ Nanomaterials have an interesting impact on various fields of application due to their desirable properties, including chemical stability, because of the large surface area-to-volume ratio, thus allowing a wide range of molecules to be coated around their surface. ${ }^{18}$ Another important property attributed to nanomaterials is their modifiable surface chemistry. Most nanomaterial platforms can be coated or functionalized with bioactive molecules to improve their 
availability, solubility and reactivity with added targeting and imaging ability when formulated correctly. ${ }^{19}$

Nanomaterials create new possibilities in cancer therapeutics discovery as their size and structures are dependent on the method of synthesis to exhibit desired physical and chemical properties. ${ }^{20}$ A review on the usage of nanomaterials for medical applications stated that nanocarriers are used to facilitate the mechanism of drug delivery to the targeted site. Nanocarriers have multiple functionality allowing them to 1) improve the hydrophilicity of anticancer drugs; ${ }^{21}$ 2) promote efficient drug delivery to tumor sites across cell barriers; ${ }^{22}$ and 3 ) enable specific site-targeted delivery of anticancer drugs with possibilities of tracking and imaging. ${ }^{23}$

Although the advantage of nanomaterials appears to be promising, it is rational that the setbacks and fundamental disadvantages are understood through more creative research studies. Among the problems and limitations of nanocarriers include their role in cellular uptakes, long-term toxicity and excretion mechanism are equally important. ${ }^{24}$ Apart from the potential toxicity of the engineered nanomaterial for medical application, another issue to consider is the biological barriers across the cell membrane. Table 1 compares and contrasts the advantages of potential nanomaterials employed for targeted cancer therapeutic drug delivery and the current clinical trial status. Most research for the breakthrough of specific drug delivery systems is only able to achieve in vivo status, especially for nanocarriers where toxicity is concerned.

Targeting of nanomaterials to specific sites is the major bottleneck in nanomedicine. ${ }^{2}$ There are two targeting strategies that have been suggested by previous researchers: active targeting and passive targeting. ${ }^{9}$ Active targeting is achieved by the binding of a high-affinity targeting ligand to the surface of the nanocarriers in order to facilitate specific cell targeting. ${ }^{25}$ There are a wide range of targeting moieties that can be used for cell-targeting purposes, including small molecules and macromolecules. In one study, folic acid (FA) was used to target cervical cancer cell line (HeLa), as FA is known to be a commonly expressed receptor by cancer cells. It was observed that the nanocarriers entered the cells via the FA receptor-mediated pathway and the drug was released within the cell. ${ }^{26}$ Passive targeting is largely dependent on the size of the nanocarriers relying on the unique characteristics of tumor blood vessel. ${ }^{25}$ Tumor sites have a large number of pores and poor lymphatic drainage, enabling passive targeting to make use of endocytosis mechanism or diffusion across the cellular membrane. ${ }^{19}$

\section{Organic-based nanomaterial}

Organic-based nanomaterials are mostly available naturally or are synthetically engineered for biomedical purposes. A wide range of organic nanocarriers are available for various applications; however, the more common type of organic nanocarriers researched for drug delivery include solid-lipid carriers, lipid carriers and polymeric carriers. ${ }^{2}$

Solid-lipid nanocarriers (SLNs) are made up of tolerant physiological lipid component, combining the advantages of polymeric nanoparticles, liposomes and fat emulsions. ${ }^{27}$ Having unique physiochemical properties makes SLNs

Table I Advantages and disadvantages of different nanomaterials for breast cancer treatment and current clinical trial status

\begin{tabular}{|c|c|c|c|}
\hline \multirow[t]{2}{*}{ Nanomaterials } & \multicolumn{2}{|l|}{ Targeted drug therapy } & \multirow{2}{*}{$\begin{array}{l}\text { Clinical trials for breast } \\
\text { cancer treatment }\end{array}$} \\
\hline & Advantages & Disadvantages & \\
\hline $\begin{array}{l}\text { Solid-lipid } \\
\text { nanoparticles } 29,30,74\end{array}$ & $\begin{array}{l}\text { - Good solubility and bioavailability due } \\
\text { to organic makeup } \\
\text { - Better control of drug release kinetic }\end{array}$ & $\begin{array}{l}\text { - Low drug loading capacities } \\
\text { - Possibly containing other } \\
\text { colloidal structures and } \\
\text { complex physical state }\end{array}$ & NA \\
\hline Liposome $e^{31,75-78}$ & $\begin{array}{l}\text { - Wide range of drug delivery } \\
\text { applications } \\
\text { - Able to increase drug load and } \\
\text { minimize undesired drug activity }\end{array}$ & $\begin{array}{l}\text { - Cationic lipids cause toxicity } \\
\text { - Rapid degradation of the } \\
\text { nanocarriers by mononuclear } \\
\text { phagocyte system (MPS) }\end{array}$ & $\begin{array}{l}\text { Liposome-annamycin Phase I/II } \\
\text { (annamycin in lipid composition } \\
\text { of DSPC, DSPG and Tween for } \\
\text { intravenous administration) }\end{array}$ \\
\hline Polymeric ${ }^{79}$ & $\begin{array}{l}\text { Versatility in terms of chemical } \\
\text { composition }\end{array}$ & Degradation of the carrier & $\begin{array}{l}\text { Nanoxel Phase I (Paclitaxel in } \\
\text { polymeric micelle) }\end{array}$ \\
\hline $\begin{array}{l}\text { Magnetic } \\
\text { nanoparticle }^{45}\end{array}$ & $\begin{array}{l}\text { Influenced by exterior magnetic field for } \\
\text { guided therapy, imaging and drug delivery }\end{array}$ & Potential material toxicity & NA \\
\hline Quantum dots ${ }^{55,80}$ & $\begin{array}{l}\text { Fluorescent properties for imaging and } \\
\text { drug tracking }\end{array}$ & Potential material toxicity & NA \\
\hline $\begin{array}{l}\text { Carbon } \\
\text { nanotubes } \\
\end{array}$ & $\begin{array}{l}\text { Able to penetrate and localize at } \\
\text { cellular level for the delivery of } \\
\text { chemotherapeutic and imaging agent }\end{array}$ & Potential material toxicity & NA \\
\hline
\end{tabular}

Abbreviations: DSPC, distearoylphophatidylcholine; DSPG, distearoylphophatidylglycerol; NA, not available. 
a potential approach for drug delivery because of their non-toxic material. ${ }^{28}$ It was previously reviewed that the advantages of SLNs include having good biocompatibility, high versatility, high drug loading efficacy and better drug release kinetics with long-term stability. ${ }^{29} \mathrm{~A}$ study was conducted using curcumin-conjugated SLN on a MCF-7 breast cancer cell line to determine its efficacy against the cancer cells, and we observed gradual drug release for 12 hours. The results showed significant cytotoxic effect compared to curcumin alone. ${ }^{30}$

Liposome nanocarriers are another potential nanocarriers. Their organic makeup benefits drug delivery application, as liposomes have high biocompatibility and less toxicity concerns. ${ }^{31}$ Liposomes have vesicle-like structures with hydrophobic lipid bilayer surrounding a water-soluble cavity, enabling both soluble and insoluble drugs to be incorporated within the core without chemical modification. Furthermore, the lipid bilayer structure on liposomes encourages fusion with other cell bilayers, promoting the release of drug content to delivery sites. ${ }^{32}$ In the past, studies have been conducted by optimizing liposomal carriers to aid the delivery of ceramide to MDA-MD-231 breast adenocarcinoma cells. The in vitro studies resulted in significant accumulation and release of ceramide in cells inducing apoptosis, proving that nanocarriers have the ability to improve drug release and delivery. ${ }^{33}$ However, SLNs are still regarded as a better carrier compared to liposomes because of their colloidal stability and biodegradability. ${ }^{32}$

Polymeric nanocarriers have a unique structure, which is basically made up of a polymer core with a center for drug loading. Polymeric carriers can be further modified to achieve multifunctional drug delivery by adding a polymer shell whose surface is modifiable to accommodate ligands and targeting moieties. ${ }^{34}$ Each polymeric nanocarrier can be uniquely designed and synthesized to exhibit desired chemistry, control release of bioactive compounds, thus giving it an added advantage of flexibility. ${ }^{35}$ A study was previously conducted by loading doxorubicin (DOX) into polymeric-lipid hybrid nanoparticle carrier to observe its loading and release efficiency and its cytotoxicity using MDR human breast cancer cell lines. The in vitro study showed a significant increase in the efficiency of DOX encapsulation with significant results for both cellular uptake and toxicity when treated with DOX-polymeric-lipid. ${ }^{36}$

In more recent reports, polymeric nanoparticles in the form of chitosan and dextran were seen to be highly popular in research. The underlying reason to this is probably due to the fact that they are naturally occurring polymers, which promote biocompatibility allowing nanomaterial toxicity to be at the least of concern. For instance, one study showed that cisplatin-loaded luteinizing hormone-releasing hormonemodified dextran nanoparticles were able to suppress breast tumor. It demonstrated not only significant antitumor effect but also antimetastasis efficacy with low systemic toxicity. ${ }^{37}$ Another study suggests the usage of peptide chains with copolymer for breast cancer-targeted HER2+ therapy. It was shown that this peptide minidrug is able to suppress cancer cell proliferation and reduce tumor size through in vivo studies due to its high affinity for HER2+ receptor binding. ${ }^{38}$

\section{Inorganic-based nanomaterial}

Inorganic nanomedicine refers to the synthesis of nanocarriers from metallic and semimetallic materials for the potential application of drug delivery. Chemically modified inorganic nanoparticles are presented as another alternative for cellular delivery breakthrough due to their versatile properties. ${ }^{39}$ These properties include controlled release, multiple functionality, good biocompatibility and capable of facilitating targeted drug delivery with imaging possibilities. ${ }^{40}$ Their unique sizedependent physicochemical properties have caused inorganic nanomaterials, such as magnetic nanoparticles (MNPs), quantum dots (QDs) and carbon nanotubes (CNTs), to gain significance in the discovery of cancer therapeutics. ${ }^{41}$

MNPs are a class of nanocarriers having iron oxide, a ferromagnetic material as part of their makeup. ${ }^{42}$ The presence of ferromagnetic material makes MNPs capable of responding to an external magnetic field when subjected to magnetic moment. ${ }^{43}$ The discovery of MNPs has attracted a lot of research interests mainly due to their ability to perform simultaneous functions, such as multimodal imaging agent for magnetic resonance imaging (MRI) and also as colloidal carriers capable of drug delivery targeted at tumor sites with real-time monitoring. ${ }^{44,45}$ The simplest form of MNPs for drug delivery consists of an inorganic material core and a modified surface coating to encourage stability and biocompatibility under physiological environments. ${ }^{46}$ Studies have been conducted previously to determine the effect of DOX-loaded magnetic poly(lactic-co-glycolic acid) nanoparticles on cancer cell lines. The results demonstrated good cytotoxicity and sensitivity toward MRI with the controlled release of anticancer drug sustained over 3 weeks. ${ }^{47}$

QDs are tiny inorganic semiconductor nanocrystals with diameter of 1-10 nm, which have the ability to fluoresce when excited by an energy source, making them attractive for biological imaging, diagnosis and therapeutic applications. ${ }^{48}$ The atomic makeup of QDs are elements of the periodic table. 
The general structure of QDs consists of a semiconductor core made up of group II-VI or group III-V elements, coated by a shell to modify their physicochemical properties and capping to promote solubility. ${ }^{49} \mathrm{QDs}$ have gained much attention in cancer research with their unique surface chemistry available for modification and multiple wavelength luminescences with high photostability, making them attractive for targeted drug delivery. ${ }^{50,51}$ Their versatility for bioconjugation with various biomolecules and imaging advantages make QDs outshine other nanocarriers. ${ }^{52,53}$

However, the major drawback of QDs lies within their toxicity and excretion pathway. Studies have shown that their toxicity is very much dependent on their core-shell material, bioconjugation and surface functionalization. ${ }^{54}$ It was reported that cadmium-based QDs are more toxic compared to non-cadmium QDs, especially for in vivo application, thus, leading to more potential studies on the synthesis of non-cadmium QDs. ${ }^{50}$ In another study, it was reported that different surface modification of QDs affects the degree of toxicity after long-term exposure together with their deposition in different organs showing the importance of QD coating. ${ }^{55}$ One study showed how QDs induced epigenetic and genotoxic changes in breast cancer cell line, MCF-7. It was observed that high dosage treatment caused cellular changes in the nucleus and mitochondria, leading to cell damage and death. ${ }^{56}$ To date, there has been not many literature regarding cytotoxicity and cellular mechanism studies of QDs with breast cancer cell lines. This is because QDs have their core and shell set made up of metallic elements causing potential toxicity. ${ }^{57}$

CNTs are made up of carbon atoms having a seamless hollow cylindrical structure from graphene sheets. ${ }^{58}$ There are two types of CNTs: single-walled nanotubes (SWNTs) and multi-walled nanotubes (MWNTs), with SWNT made up of a single sheet of graphene and MWNT made up of multilayers of graphene sheets. ${ }^{59}$ Since the discovery of CNTs, both SWNT and MWNT have attracted much attention due to their promising size, needle-like structure and unique physicochemical properties, thus, making them ideal for drug delivery systems. ${ }^{60}$ Although CNTs are said to be hydrophobic in nature as they are a carbon family of fullerenes, their rich surface chemistry allows functionalization with hydrophilic biomolecules, drugs, proteins or ligands to increase their solubility and biocompatibility while decreasing their cytotoxicity. ${ }^{39}$ There are three possible applications of CNT-drug interaction, including absorption of the active compound into CNT mesh, covalent or noncovalent attachment of biomolecules onto the walls of CNTs and as catheters utilizing the CNT channels. ${ }^{41} \mathrm{CNT}$ s are seen as more promising nanocarriers for cancer because of their high drug loading efficacy, cell membrane penetration capability and $\mathrm{pH}$-dependency on their drug release. ${ }^{61}$

Nevertheless, there are concerns for the application of CNTs for drug delivery systems including their hydrophobicity and cytotoxicity. CNTs are known to require purification and modification due to the presence of contaminants during synthesis processes, causing them to be insoluble, toxic and unavailable to biological systems. ${ }^{62}$ Research conducted previously have concluded that CNTs that have been purified and modified hold a higher potential for targeted drug delivery systems, since their material encapsulation is able to overcome the limitations posed by CNTs themselves and also the barriers of conventional therapeutic strategies. ${ }^{63}$ One study particularly demonstrated the use of only cationic polymer with CNT to evaluate its cytotoxicity and cellular uptake on breast cancer cell line, MCF-7. It was found that the CNT itself was toxic, and hence, excluded from cell penetration but modified CNT appeared to be less toxic to cells and was found to localize in the cytoplasm. ${ }^{64}$

\section{Encapsulation of anti-breast cancer drugs with nanomaterials}

The development of nanotechnology has brought about endless active research of various nanomaterials and their drug conjugates as potential nanocarriers to pursue successful drug delivery to breast cancer sites. Both organic and inorganic nanostructures alike show great potential for clinical applications and are taking a lead in the discovery of cancer breakthrough. ${ }^{65}$ Nanomaterials are particularly interesting for drug delivery mostly due to their enhanced drug loading efficiency and ability to internalize at cellular level due to their nanosize. ${ }^{50}$ A significant number of ongoing studies have demonstrated the use of different nanomaterials with different drugs and their positive activity toward breast cancer as effective drug delivery systems. Table 2 shows a summary of the various nanomaterials with drug conjugate for anti-breast cancer studies.

The type of administered therapeutic drugs for individual breast cancer diagnosis is different depending on the protein biomarker expression. ${ }^{66}$ The type of biomarker present on the cancer cells determines the type of drug administration since its cell classification and immunoprofile are bound to be different. ${ }^{67}$ The following section briefly discusses the toxicology profile and biodistribution of the nanocarriers conjugated with various therapeutic drugs targeted for breast cancer. 


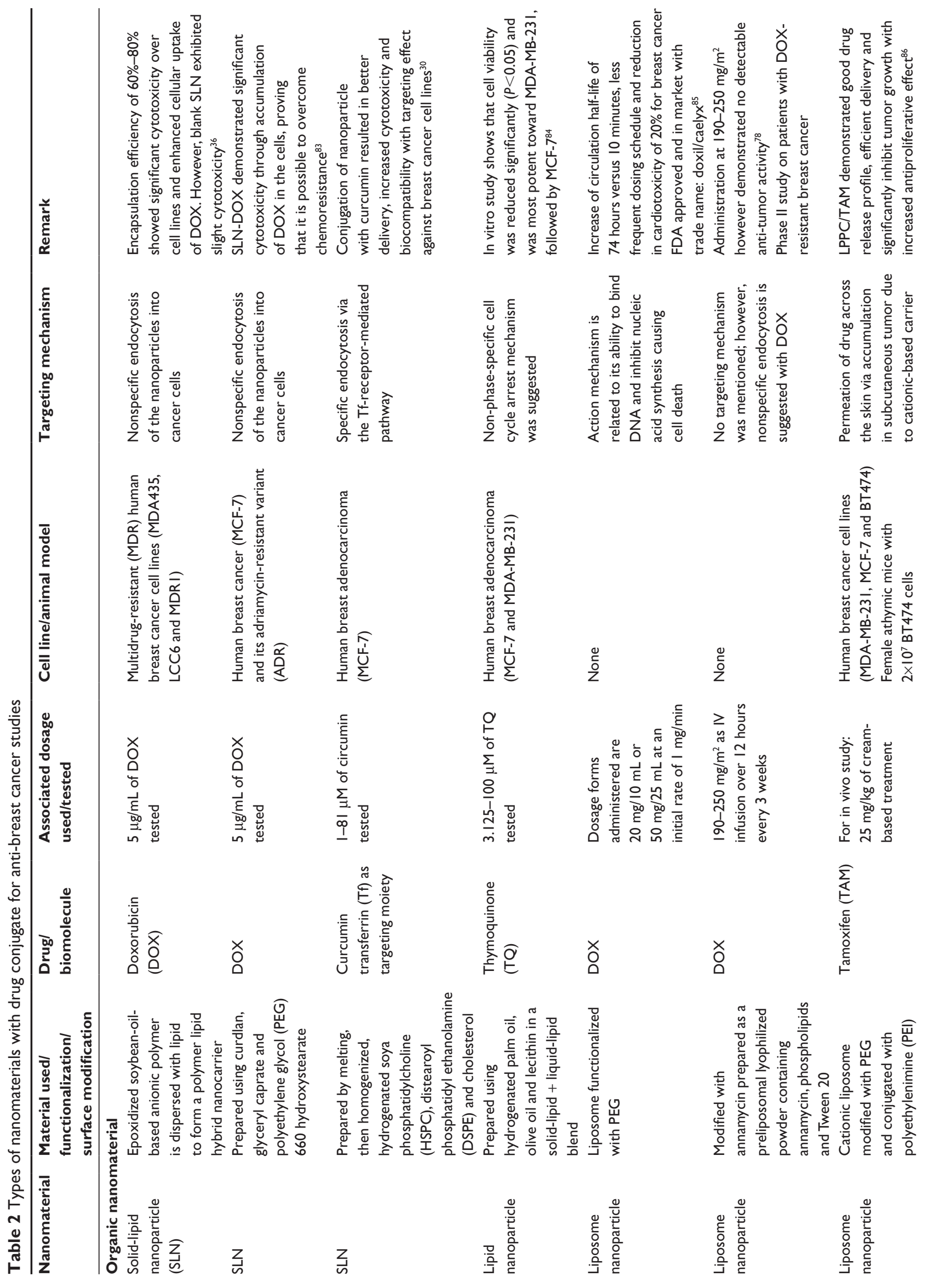




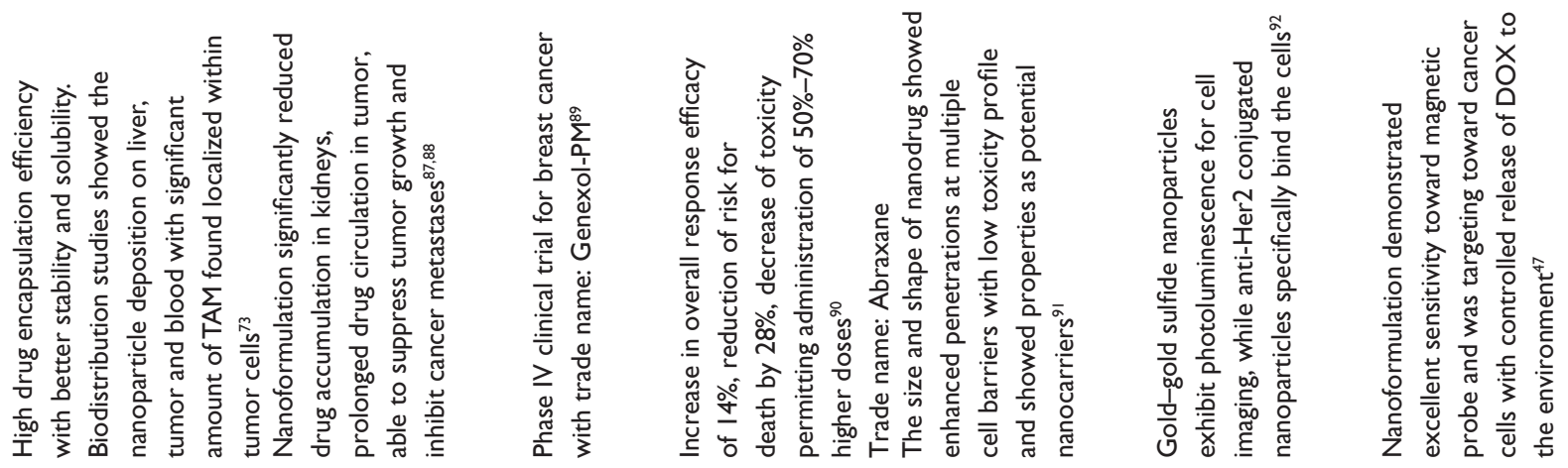
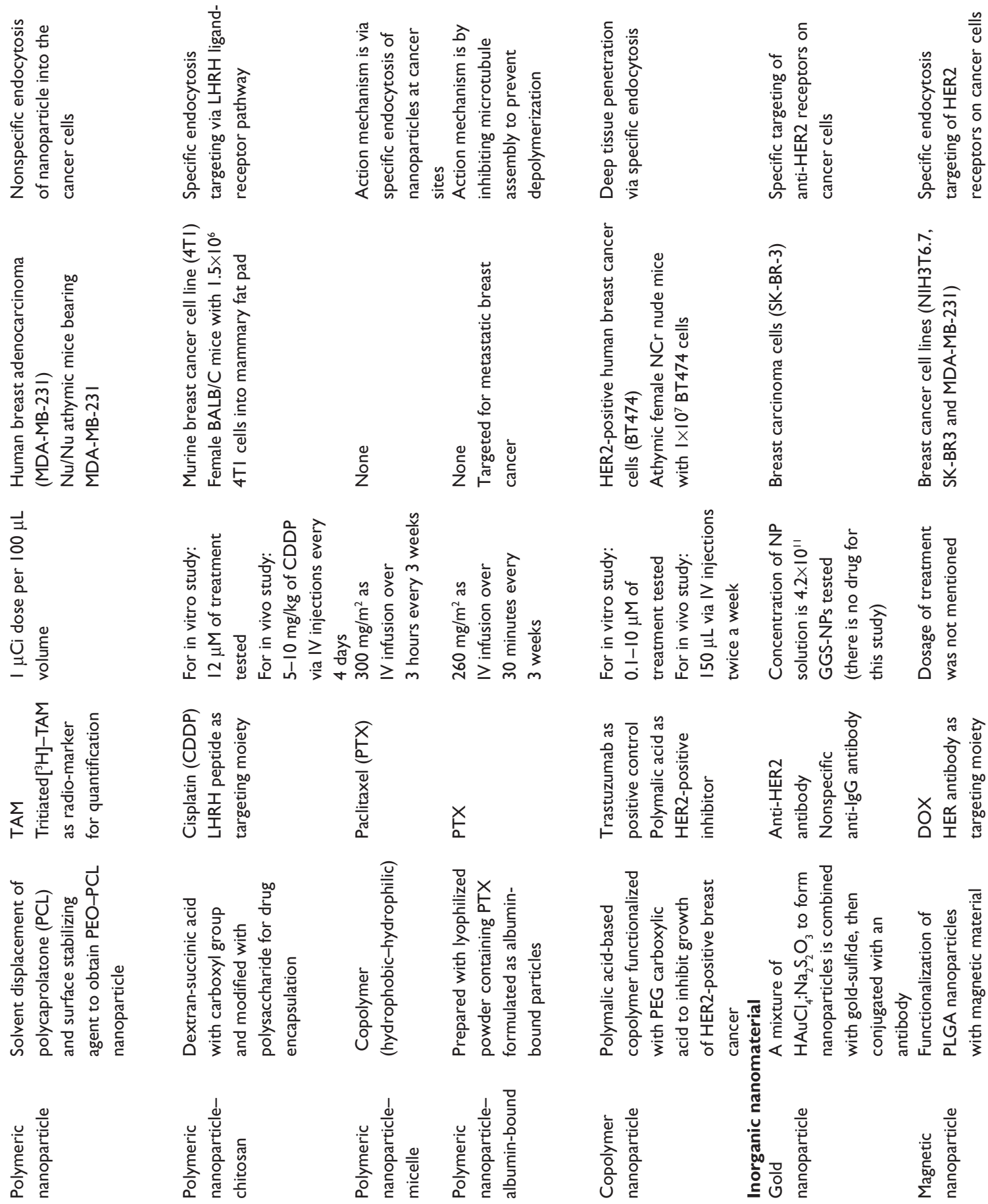

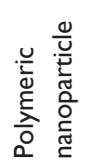
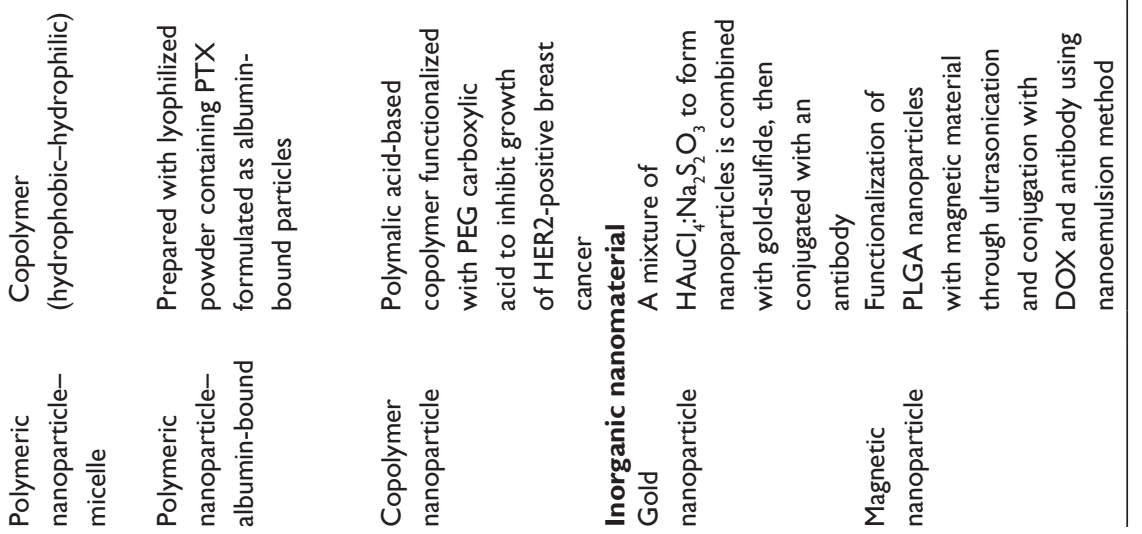


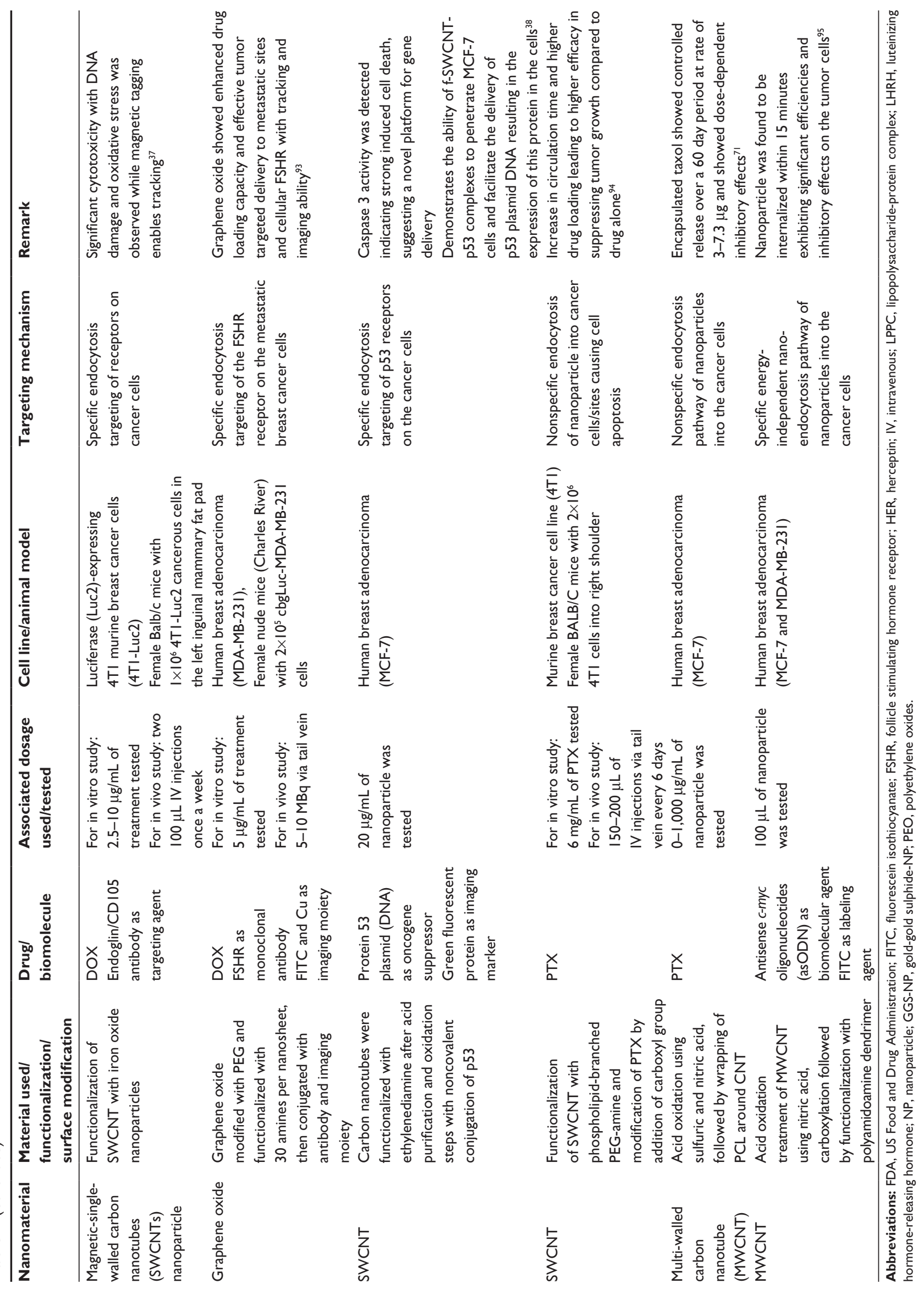


Common chemotherapy drug category for the treatment of breast cancer includes anthracyclines, taxanes and cyclophosphamide. Anthracyclines are a group of anticancer drugs whose DNA interaction causes antineoplastic effects. This interaction results in DNA damage triggering apoptosis in cells. They are effective against cancerous cells but their toxicity does not discriminate between malignant and healthy cells. ${ }^{68}$ DOX belongs to the group of anthracyclines that is used to treat breast cancer. However, its limited half-life and high toxicity of affecting healthy cells make DOX limited for clinical application. Owing to this factor, various studies were carried out through the years to allow DOX to be attached to a carrier in order to increase its efficacy and reduce side effects. ${ }^{69}$ To further investigate the effect of DOX conjugated with a nanocarrier, one study has developed a nanoformulation containing DOX and functionalized MWNT. The nanoformulation was studied using an MCF-7 human breast cancer cell line and was found to be effective in inhibiting cell growth by inducing apoptosis. It was also shown that the nanoformulation mostly targeted cancerous cells through the receptor-mediated endocytosis mechanism. ${ }^{70}$

Taxanes is another group of chemotherapy drugs used for breast cancer in the early stage. They prevent the growth of cancer cells by disrupting microtubules from breaking down into cancer cell structures, so they cannot grow and divide. ${ }^{68}$ An example of taxanes is paclitaxel (PTX) that is also commonly used for the treatment of metastatic breast cancer. However, similar to other drawbacks of commercially available drugs, PTX lacks efficient distribution, selectivity and solubility. There was research conducted previously on the conjugation of PTX with a carbon-based nanomaterial to study its cytotoxicity on MCF-7 breast cancer cell line. The conjugated PTX showed a dose-dependent inhibitory effect with better release rate. ${ }^{71}$

Cyclophosphamide is used to treat women, who do not have hormone receptors or have high probability for relapse..$^{68}$ While tamoxifen (TAM) is a non-steroidal anti-estrogen that is long used for the treatment of post-menopausal breast cancer patients. There was a significant effect using TAM on the treatment of hormone receptor-positive breast cancer. It was believed that the main inhibitory mechanism for TAM is related to the estrogen receptor but researchers have demonstrated the possible existence of non-estrogen receptor mechanism as well. ${ }^{72} \mathrm{~A}$ study was conducted previously to evaluate the distribution profile of TAM in polymeric nanoparticles on human breast carcinoma xenograft, MDA-MB-231. A high TAM encapsulation efficiency was achieved and the nanoformulation showed better stability, and a significant amount of TAM was found to internalize tumor cells. ${ }^{73}$

\section{Conclusion}

The future of nanomedicine has become an interesting debate. As a result, many biocompatible nanomaterials have been researched, conjugated with various anti-breast cancer drugs, and their potential toxicity and possible cellular mechanisms have been studied. Although there are a few organic-based nanomaterials that have managed to enter clinical trials, there are still a lot of research to be conducted to fully understand the full potential and drawbacks of the nanoformulations. Nanomaterials may show remarkable advantages for a perfect drug delivery system due to their ability for functionalization to render tracking, imaging and targeting properties, but there are still much to consider, especially for in vivo application toward clinical trials. This is because nanomaterials are naturally non-immunogenic owing to their nanosize, and therefore, show great carrier features with a tendency to by-pass cellular membranes. Therefore, a longer time frame will be needed for the study of material excretion and long-term toxicity. Even though there are still many uncertainties associated with the toxicity and uptake of these nanomaterials, the current findings described strongly prove that nanomaterials hold a great future toward the field of nanomedicine for breast cancer treatment.

\section{Disclosure}

The authors report no conflicts of interest in this work.

\section{References}

1. Madani SY, Naderi N, Dissanayake O, Tan A, Seifalian AM. A new era of cancer treatment: carbon nanotubes as drug delivery tools. Int J Nanomedicine. 2011;6:2963-2979.

2. Wicki A, Witzigmann D, Balasubramanian V, Huwyler J. Nanomedicine in cancer therapy: challenges, opportunities, and clinical applications. J Control Release. 2015;200:138-157.

3. DeSantis C, Siegel R, Bandi P, Jemal A. Breast cancer statistics, 2011. CA Cancer J Clin. 2011;61(6):409-418.

4. Torre LA, Bray F, Siegel RL, Ferlay J, Lortet-Tieulent J, Jemal A. Global cancer statistics, 2012. CA Cancer J Clin. 2015;65(2):87-108.

5. Probst CE, Zrazhevskiy P, Bagalkot V, Gao X. Quantum dots as a platform for nanoparticle drug delivery vehicle design. Adv Drug Deliv Rev. 2013;65(5):703-718.

6. Wang Y, Chen L. Quantum dots, lighting up the research and development of nanomedicine. Nanomedicine. 2011;7(4):385-402.

7. Couvreur P. Nanoparticles in drug delivery: past, present and future. $A d v$ Drug Deliv Rev. 2013;65(1):21-23.

8. Lehner R, Wang X, Marsch S, Hunziker P. Intelligent nanomaterials for medicine: carrier platforms and targeting strategies in the context of clinical application. Nanomedicine. 2013;9(6):742-757.

9. Grobmyer SR, Zhou G, Gutwein LG, Iwakuma N, Sharma P, Hochwald SN. Nanoparticle delivery for metastatic breast cancer. Maturitas. 2012;73(1): 19-26. 
10. Slamon DJ, Leyland-Jones B, Shak S, et al. Use of chemotherapy plus a monoclonal antibody against HER2 for metastatic breast cancer that overexpresses HER2. $N$ Engl J Med. 2001;344(11): 783-792.

11. Cancerresearch.org [homepage on the Internet]. New York: Cancer Research Institute [updated 2016]. Available from: http://www. cancerresearch.org/Cancer-Immunotherapy. Accessed June 28, 2016.

12. Cancer.gov [homepage on the Internet]. United States of America: National Institute of Health [updated 2016]. Available from: https:// www.cancer.gov/types/breast/patient/breast-treatment-pdq. Accessed June 28, 2016.

13. Batra R, Davies JN, Wheatley D. Extensive arterial and venous thromboembolism with chemotherapy for testicular cancer: a case report. Cases J. 2009;2:9082.

14. Chomoucka J, Drbohlavova J, Huska D, Adam V, Kizek R, Hubalek J. Magnetic nanoparticles and targeted drug delivering. Pharmacol Res. 2010;62(2):144-149.

15. Chidambaram M, Manavalan R, Kathiresan K. Nanotherapeutics to overcome conventional cancer chemotherapy limitations. $J$ Pharm Pharm Sci. 2011;14(1):67-77.

16. Bikiaris D, Papageorgiou GZ, Stergiou A, et al. Physicochemical studies on solid dispersions of poorly water-soluble drugs: evaluation of capabilities and limitations of thermal analysis techniques. Thermochim Acta. 2005;439(1-2):58-67.

17. Liu BR, Winiarz JG, Moon JS, et al. Synthesis, characterization and applications of carboxylated and polyethylene-glycolated bifunctionalized $\mathrm{InP} / \mathrm{ZnS}$ quantum dots in cellular internalization mediated by cell-penetrating peptides. Colloids Surf B Biointerfaces. 2013; 111:162-170.

18. Yamashita T, Yamashita K, Nabeshi H, et al. Carbon nanomaterials: efficacy and safety for nanomedicine. Materials. 2012;5(2):350-363.

19. Bamrungsap S, Zhao Z, Chen T, et al. Nanotechnology in therapeutics: a focus on nanoparticles as a drug delivery system. Nanomedicine (Lond). 2012;7(8):1253-1271.

20. Bystrov VS, Bdikin IK, Heredia A, et al. Piezoelectricity and Ferroelectricity in Biomaterials: From Proteins to Self-assembled Peptide Nanotubes. In: Ciofani G, Menciassi A, editors. Piezoelectric Nanomaterials for Biomedical Applications. Heidelberg: Springer; 2012:187.

21. Cao X, Deng W, Fu M, et al. Seventy-two-hour release formulation of the poorly soluble drug silybin based on porous silica nanoparticles: in vitro release kinetics and in vitro/in vivo correlations in beagle dogs. Eur J Pharm Sci. 2013;48(1-2):64-71.

22. Ghaffarian R, Bhowmick T, Muro S. Transport of Nanocarriers across Gastrointestinal Epithelial cells by a New Transcellular Route Induced by Targeting ICAM-1. J Control Release. 2012;163(1):25-33.

23. Zhang L, Xue H, Cao Z, Keefe A, Wang J, Jiang S. Multifunctional and degradable zwitterionic nanogels for targeted delivery, enhanced MR imaging, reduction-sensitive drug release, and renal clearance. Biomaterials. 2011;32(20):4604-4608.

24 . Nie S. Understanding and overcoming major barriers in cancer nanomedicine. Nanomedicine (Lond). 2010;5(4):523-528.

25. Alexis F, Pridgen EM, Langer R, Farokhzad OC. Nanoparticle technologies for cancer therapy. Handb Exp Pharmacol. 2010;(197):55-86.

26. Zhang X, Meng L, Lu Q, Fei Z, Dyson PJ. Targeted delivery and controlled release of doxorubicin to cancer cells using modified single wall carbon nanotubes. 2009;30(30):6041-6047.

27. Hu L, Tang X, Cui F. Solid lipid nanoparticles (SLNs) to improve oral bioavailability of poorly soluble drugs. J Pharm Pharmacol. 2004; 56(12):1527-1535.

28. Almeida AJ, Souto E. Solid lipid nanoparticles as a drug delivery system for peptides and proteins. Adv Drug Deliv Rev. 2007;59(6):478-490.

29. Ekambaram P, Sathali AA, Priyanka K. Solid lipid nanoparticles: a review. Sci Revs Chem Commun. 2012;2(1):80-102.

30. Mulik RS, Mönkkönen J, Juvonen RO, Mahadik KR, Paradkar AR. Transferrin mediated solid lipid nanoparticles containing curcumin: enhanced in vitro anticancer activity by induction of apoptosis. Int $J$ Pharm. 2010;398(1-2):190-203.
31. Caracciolo G. Liposome-protein corona in a physiological environment: challenges and opportunities for targeted delivery of nanomedicines. Nanomedicine. 2015;11(3):543-557.

32. Mudshinge SR, Deore AB, Patil S, Bhalgat CM. Nanoparticles: emerging carriers for drug delivery. Saudi Pharm J. 2011;19(3):129-141.

33. Stover T, Kester M. Liposomal delivery enhances short-chain ceramideinduced apoptosis of breast cancer cells. J Pharmacol Exp Ther. 2003; 307(2):468-475.

34. Prakash S, Malhotra M, Shao W, Tomaro-Duchesneau C, Abbasi S. Polymeric nanohybrids and functionalized carbon nanotubes as drug delivery carriers for cancer therapy. Adv Drug Deliv Rev. 2011;63(14-15): 1340-1351

35. Yang D, Yu L, Van S. Clinically relevant anticancer polymer paclitaxel therapeutics. Cancers (Basel). 2010;3(1):17-42.

36. Wong HL, Rauth AM, Bendayan R, et al. A new polymer-lipid hybrid nanoparticle system increases cytotoxicity of doxorubicin against multidrug-resistant human breast cancer cells. Pharm Res. 2006;23(7): 1574-1585.

37. Al Faraj A, Shaik AP, Shaik AS. Magnetic single-walled carbon nanotubes as efficient drug delivery nanocarriers in breast cancer murine model: noninvasive monitoring using diffusion-weighted magnetic resonance imaging as sensitive imaging biomarker. Int J Nanomedicine. 2014;10:157-168.

38. Karmakar A, Bratton SM, Dervishi E, et al. Ethylenediamine functionalized-single-walled nanotube (f-SWNT)-assisted in vitro delivery of the oncogene suppressor p53 gene to breast cancer MCF-7 cells. Int $J$ Nanomedicine. 2011;6:1045-1055.

39. Xu ZP, Zeng QH, Lu GQ, Yu AB. Inorganic nanoparticles as carriers for efficient cellular delivery. Chem Eng Sci. 2006;61(3):1027-1040.

40. Bauer LA, Birenbaum NS, Meyer GJ. Biological applications of high aspect ratio nanoparticles. J Mater Chem. 2004;14(4):517-526.

41. Huang H, Yuan Q, Shah JS, Misra RD. A new family of folate-decorated and carbon nanotube-mediated drug delivery system: synthesis and drug delivery response. Adv Drug Deliv Rev. 2011;63(14-15):1332-1339.

42. Soenen SJ, Rivera-Gil P, Montenegro JM, Parak WJ, De Smedt SC, Braeckmans K. Cellular toxicity of inorganic nanoparticles: common aspects and guidelines for improved nanotoxicity evaluation. Nano Today. 2011;6(5):446-465.

43. Mornet S, Vasseur S, Grasset F, Duguet E. Magnetic nanoparticle design for medical diagnosis and therapy. J Mater Chem. 2004;14(14): 2161-2175.

44. Frullano L, Meade TJ. Multimodal MRI contrast agents. J Biol Inorg Chem. 2007;12(7):939-949

45. Pankhurst QA, Connolly J, Jones SK, Dobson JJ. Applications of magnetic nanoparticles in biomedicine. J Phys D Appl Phys. 2003; 36(13):R167-R181.

46. McNeil SE. Nanotechnology for the biologist. J Leukoc Biol. 2005; 78(3):585-594

47. Yang J, Lee CH, Park J, et al. Antibody conjugated magnetic PLGA nanoparticles for diagnosis and treatment of breast cancer. $J$ Mater Chem. 2007;17(26):2695-2699.

48. Modani SH, Kharwade ME, Nijhawan MO. Quantum dots: a novelty of medical field with multiple applications. Int J Curr Pharm Res. 2013; 5(4):55-59.

49. Sekhon BS, Kamboj SR. Inorganic nanomedicine - part 1. Nanomedicine. 2010;6(4):516-522.

50. Yong KT, Wang Y, Roy I, et al. Preparation of quantum dot/drug nanoparticle formulations for traceable targeted delivery and therapy. Theranostics. 2012;2(7):681-694.

51. Surana K, Singh PK, Rhee HW, Bhattacharya B. Synthesis, characterization and application of CdSe quantum dots. J Ind Eng Chem. 2014; 20(6):4188-4193.

52. Zhu H, Hu MZ, Shao L, et al. Synthesis and optical properties of thiol functionalized $\mathrm{CdSe} / \mathrm{ZnS}$ (core/shell) quantum dots by ligand exchange. J Nanomaterials. 2014;2014:1-14.

53. Smith AM, Duan H, Mohs AM, Nie S. Bioconjugated quantum dots for in vivo molecular and cellular imaging. Adv Drug Deliv Rev. 2008; 60(11):1226-1240. 
54. Bradburne CE, Delehanty JB, Boeneman Gemmill K, et al. Cytotoxicity of quantum dots used for in vitro cellular labeling: role of QD surface ligand, delivery modality, cell type, and direct comparison to organic fluorophores. Bioconjug Chem. 2013;24(9):1570-1583.

55. Tang Y, Han S, Liu H, et al. The role of surface chemistry in determining in vivo biodistribution and toxicity of $\mathrm{CdSe} / \mathrm{ZnS}$ core-shell quantum dots. Biomaterials. 2013;34(34):8741-8755.

56. Choi AO, Brown SE, Szyf M, Maysinger D. Quantum dot-induced epigenetic and genotoxic changes in human breast cancer cells. $J \mathrm{Mol}$ Med. 2008;86(3):291-302.

57. Kuzyniak W, Adegoke O, Sekhosana K, et al. Synthesis and characterization of quantum dots designed for biomedical use. Int J Pharm. 2014; 466(1-2):382-389.

58. Ganesh EN. Single walled and multi walled carbon nanotube structure, synthesis and applications. Int J Innovative Technology Exploring Engineering. 2013;2(4):311-320.

59. Liu Z, Tabakman S, Welsher K, Dai H. Carbon nanotubes in biology and medicine: in vitro and in vivo detection, imaging and drug delivery. Nano Res. 2009;2(2):85-120.

60. Heister E, Neves V, Tîlmaciu C, et al. Triple functionalisation of singlewalled carbon nanotubes with doxorubicin, a monoclonal antibody, and a fluorescent marker for targeted cancer therapy. Carbon. 2009; 47(9):2152-2160.

61. Ji Z, Lin G, Lu Q, et al. Targeted therapy of SMMC-7721 liver cancer in vitro and in vivo with carbon nanotubes based drug delivery system. J Colloid Interface Sci. 2012;365(1):143-149.

62. Prato M, Kostarelos K, Bianco A. Functionalized carbon nanotubes in drug. 2008;41(1):60-68

63. Eatemadi A, Daraee H, Karimkhanloo H, et al. Carbon nanotubes: properties, synthesis, purification, and medical applications. Nanoscale Res Lett. 2014;9(1):393.

64. Pan BF, Cui DX, Xu P, et al. Cellular uptake enhancement of polyamidoamine dendrimer modified single walled carbon nanotubes. Biomed Pharma Eng. 2007;1:13-16

65. Webster DM, Sundaram P, Byrne ME. Injectable nanomaterials for drug delivery: carriers, targeting moieties, and therapeutics. Eur $J$ Pharm Biopharm. 2013;84(1):1-20.

66. Yezhelyev MV, Gao X, Xing Y, Al-Hajj A, Nie S, O’Regan RM. Emerging use of nanoparticles in diagnosis and treatment of breast cancer. Lancet Oncol. 2006;7(8):657-667.

67. Holliday DL, Speirs V. Choosing the right cell line for breast cancer research. Breast Cancer Res. 2011;13(4):215.

68. Ligresti G, Libra M, Militello L, et al. Breast cancer: molecular basis and therapeutic strategies (review). Mol Med Rep. 2008;1(4):451-458.

69. Tan JM, Arulselvan P, Fakurazi S, Ithnin H, Hussein MZ. A review on characterizations and biocompatibility of functionalized carbon nanotubes in drug delivery design. J Nanomater. 2014;2014(1):1-20.

70. Mehra NK, Verma AK, Mishra PR, Jain NK. The cancer targeting potential of D- $\alpha$-tocopheryl polyethylene glycol 1000 succinate tethered multi walled carbon nanotubes. Biomaterials. 2014;35(15):4573-4588.

71. Kim SY, Hwang JY, Seo JW, Shin US. Production of CNT-taxolembedded PCL microspheres using an ammonium-based room temperature ionic liquid: as a sustained drug delivery system. J Colloid Interface Sci. 2015;442:147-153.

72. Ali S, Buluwela L, Coombes RC. Antiestrogens and their therapeutic applications in breast cancer and other diseases. Annu Rev Med. 2011; 62:217-232.

73. Shenoy DB, Amiji MM. Poly(ethylene oxide)-modified poly(epsiloncaprolactone) nanoparticles for targeted delivery of tamoxifen in breast cancer. Int J Pharm. 2005;293(1-2):261-270.

74. Mukherjee S, Ray S, Thakur RS. Solid lipid nanoparticles: a modern formulation approach in drug delivery system. Indian J Pharm Sci. 2009; 71(4):349-358.

75. Fan Y, Zhang Q. Development of liposomal formulations: from concept to clinical investigations. Asian J Pharm Sci. 2013;8(2):81-87.
76. Lappalainen K, Jääskeläinen I, Syrjänen K, Urtti A, Syrjänen S. Comparison of cell proliferation and toxicity assays using two cationic liposomes. Pharm Res. 1994;11(8):1127-1131.

77. Kircheis R, Schüller S, Brunner S, et al. Polycation-based DNA complexes for tumor-targeted gene delivery in vivo. J Gene Med. 1999; 1(2):111-120.

78. Booser DJ, Esteva FJ, Rivera E, et al. Phase II study of liposomal annamycin in the treatment of doxorubicin-resistant breast cancer. Cancer Chemother Pharmacol. 2002;50(1):6-8.

79. Madaan A, Singh P, Awasthi A, et al. Efficiency and mechanism of intracellular paclitaxel delivery by novel nanopolymer-based tumortargeted delivery system, Nanoxel(TM). Clin Transl Oncol. 2013; 15(1):26-32.

80. Lin G, Ouyang Q, Hu R, et al. In vivo toxicity assessment of noncadmium quantum dots in BALB/c mice. Nanomedicine. 2015;11(2): 341-350.

81. Ciobotaru CC, Damian CM, Polosan S, Prodana M, Iovu H. Drug delivery study of single-wall carbon nanotubes covalent functionalized with cisplatin. Dig J Nanomater Biostruct. 2014;9(2):859-868.

82. Firme CP 3rd, Bandaru PR. Toxicity issues in the application of carbon nanotubes to biological systems. Nanomedicine. 2010;6(2):245-256.

83. Kang KW, Chun MK, Kim O, et al. Doxorubicin-loaded solid lipid nanoparticles to overcome multidrug resistance in cancer therapy. Nanomedicine. 2010;6(2):210-213.

84. Ng WK, Saiful Yazan L, Yap LH, Wan Nor Hafiza WA, How CW, Abdullah R. Thymoquinone-loaded nanostructured lipid carrier exhibited cytotoxicity towards breast cancer cell lines (MDA-MB-231 and MCF-7) and cervical cancer cell lines (HeLa and SiHa). Biomed Res Int. 2015;2015:263131.

85. Doxil ${ }^{\circledR}$ (doxorubicin hydrochoride liposome injection), for intravenous use [prescribing information]. Unites States: Janssen Products, LP; 2010.

86. Lin YL, Chen $\mathrm{CH}, \mathrm{Wu} \mathrm{HY}$, et al. Inhibition of breast cancer with transdermal tamoxifen-encapsulated lipoplex. J Nanobiotechnology. 2016;14:11.

87. Li M, Tag Z, Zhang Y, Lv S, Li Q, Chen X. Targeted delivery of cisplatin by LHRH-peptide conjugated dextran nanoparticles suppresses breast cancer growth and metastasis. Acta Biomater. 2015;18: $132-143$.

88. Li M, Tang Z, Zhang Y, et al. LHRH-peptide conjugated dextran nanoparticles for targeted delivery of cisplatin to breast cancer. JMater Chem B. 2014;2(22):3490-3499.

89. Samyang Biopharmaceuticals Corporation. Genexol-PM (paclitaxel). Available from: http:/www.biokorea.org/upload_2013/download. asp?fn=20130620111137_003228.pdf\&fd=TECH. Accessed November 29, 2016.

90. Abraxane ${ }^{\mathbb{}}$ for injectable suspensions (paclitaxel protein-bound particles for injectable suspension) [prescribing information]. United States: Celgene Corporation; 2015.

91. Ding H, Gangalum PR, Galstyan A, et al. HER2-positive breast cancer targeting and treatment by a peptide-conjugated mini nanodrug. Nanomedicine. 2016;13(2):631-639.

92. Day ES, Bickford LR, Slater JH, Riggall NS, Drezek RA, West JL. Antibody-conjugated gold-gold sulfide nanoparticles as multifunctional agents for imaging and therapy of breast cancer. Int $J$ Nanomedicine. 2010;5:445-454.

93. Yang D, Feng L, Dougherty CA, et al. In vivo targeting of metastatic breast cancer via tumor vasculature-specific nano-graphene oxide. Biomaterials. 2016;104:361-371.

94. Liu Z, Chen K, Davis C, et al. Drug delivery with carbon nanotubes for in vivo cancer treatment. 2008;68(16):6652-6660.

95. Pan B, Cui D, Xu P, et al. Synthesis and characterization of polyamidoamine dendrimer-coated multi-walled carbon nanotubes and their application in gene delivery systems. Nanotechnology. 2009; 20(12):125101. 


\section{Publish your work in this journal}

The International Journal of Nanomedicine is an international, peerreviewed journal focusing on the application of nanotechnology in diagnostics, therapeutics, and drug delivery systems throughout the biomedical field. This journal is indexed on PubMed Central,

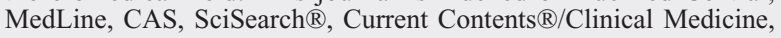

Journal Citation Reports/Science Edition, EMBase, Scopus and the Elsevier Bibliographic databases. The manuscript management system is completely online and includes a very quick and fair peer-review system, which is all easy to use. Visit http://www.dovepress.com/ testimonials.php to read real quotes from published authors.

Submit your manuscript here: http://www.dovepress.com/international-journal-of-nanomedicine-journal 